\title{
Efficient computation of viscoplasticity based on numerical model reduction with error control
}

\author{
Daniel Hård*, Henrik Svensson*, Fredrik Ekre*, Ralf Jänicke*, Fredrik Larsson*, and \\ Kenneth Runesson* \\ * Chalmers University of Technology \\ Department of Industrial and Material Sciences \\ SE-412 96 Gothenburg, Sweden \\ web page: https://www.chalmers.se/en/departments/ims/
}

\begin{abstract}
The concept of numerical model reduction (NMR) is associated with the aim to reduce the computational cost when a similar problem is solved numerous times for slightly different data. A prominent example is the solution of RVE-problems in computational homogenization. In this contribution we (re)consider the problem of small strain viscoplasticity, which may serve as a prototype for a larger class of nonlinear problems where it is possible to use the concept of Nonuniform Transformation Field Analysis (NFTA).

Using POD-modes, we investigate various strategies for low cost control of the NMR-error. In particular, we extend a recently published approach for transient linear problems, cf. Ekre et al. [1]. However, while it is possible to obtain guaranteed bounds for the linear problem, one can only hope for good estimates in the present nonlinear case. The ultimate goal is, based on the error assessment, to make the optimal refinement of the NMR-basis in the sense that modes are invoked sequentially in a hierarchical fashion.
\end{abstract}

\section{REFERENCES}

[1] Ekre F., Larsson F., Runesson K. and Jänicke R. A posteriori error estimation for numerical model reduction in computational homogenization of porous media. International Journal for Numerical Methods in Engineering (2020). 